\title{
Double Allocation of Spreading Code Minimizing SI and PAPR for LP- OFDM UWB System
}

Naziha $\mathbf{N}^{*}$ and Bouallegue $\mathbf{R}$

Innov'COM Lab, Higher School of Communications of Tunis, Sup'Com, University 7th November at Carthage, Tunisia

\begin{abstract}
Self-interference (SI) occurs if the transmission is through a multipath channel. The channel distorts helps to break the orthogonality between the spreading codes. In addition, the amplitude of linear precoded orthogonal frequency division multiplexing (LP-OFDM). LP-OFDM signals have strong fluctuations. Thus, it is necessary to seek or to minimize these two problems by the reduction of term SI and the amplitude of fluctuations Peak-to-Average Power Ratio (PAPR). In this paper a new method of allocation of spreading code has proposed. It consists of a double selection of spreading codes minimizing joint SI and PAPR. Simulation results show that the LP-OFDM system is optimized by this proposal compared to the conventional solution where the systems don't operate in full load.
\end{abstract}

Keywords: LPOFDM; Linear Precoding; spreading codes; SI; PAPR

\section{Introduction}

To ensure a satisfactory quality of service, future communications systems require a high spectral efficiency and flexibility. In 1993, many studies have shown that the technical meeting in places spread spectrum and multicarrier modulation is a solution which meets these criteria [1]. Multicarrier transmission is a solution which is widely exploited in communication networks, whether local, cellular, wireline, embedded or television [2].

The resources allocation is a fundamental aspect in the design of multicarrier systems. So, with the development of powerline communications, this theme of resource allocation in orthogonal frequency division multiplexing (OFDM) systems is still relevant [3] for access networks, home networks as embedded networks because it can maximize either the throughput or the robustness of the system [4].

The LP-OFDM systems can be described as multicarrier systems using a linear precoding and modulating data to be transmitted. The LP-OFDM is based on conventional OFDM associated with a linear precoding component. The main aim of this system is to make the most flexible multicarrier system with lowered limitations and better overall system performance, without raising its complexity [4].

In mobile radio and especially in LP-OFDM system, this precoding provides additional flexibility, simplifies the separation of received signals, or makes it possible, and improves the performance of communications systems. One of the advantages of this precoding is to allow the operation of subcarriers with low signal-to-noise ratio (SNR) [5].

In this paper, a new technique for dynamic resource allocation for LP-OFDM applications has proposed. The Exploitation of the dynamic allocation for ultra wideband (UWB) systems is a real advantage because the UWB indoor channel varies slowly over time, which reduces the system complexity.

Indeed, the proposing solution deals with the selection of spreading sequences applied to LP-OFDM signals which are based on several criteria such as correlation functions. In fact, the aim is to propose the selection of optimum spreading codes according to these criteria by minimizing jointly the SI and PAPR. Furthermore, this optimized allocation procedure can increase significantly the mean BER of the system.

This paper is organized as follows. In Section 2, the considered LPOFDM system is described. Section 3 presents the different selection criteria. In Section 4, allocation algorithm is proposed for LP-OFDM systems to reduce jointly SI and PAPR. In section 5, simulation results are discussed and presented in terms of bit error rate. Conclusions are drawn in Section 6

\section{System Description}

The insertion of a linear precoding function in the conventional OFDM system leads to a LP-OFDM system. In this particular form, the term LP-OFDM may also be referred to the Spectrum multicarrier multiple-access (SS-MC-MA) in mobile radio communications [6]

So, the system complexity is not increased significantly with the addition of precoding function. The linear precoding component may be exploited to decrease PAPR of OFDM systems [7]. The linear precoding component makes the communication more robust against the channel selectivity, and provides natural system robustness against narrowband interferers. It provides a finer granularity in the selection of transmission rates.

In the studied LP-OFDM system, each sub-band occupied by a piconet is divided into several sub-blocks. Each one comtains a number of subcarriers equal to the length $\mathrm{L}$ of the spreading codes in Figure 1.

\section{Expressions of LP-OFDM Signals}

The linear precoding operation is performed before the OFDM modulation. It can be written as

$$
\underset{N \times N_{s}}{S}=F_{N}^{H} \underset{N \times L}{D} \underset{L \times N_{L}}{C} \underset{N_{L} \times N_{s}}{X}
$$

Where $\mathrm{S}$ is the matrix of Ns OFDM symbols, each one is composed of $\mathrm{N}$ time samples. $\mathrm{X}$ is the matrix of BICM symbols to be transmitted. $\mathrm{C}$ is the linear precoding matrix. $\mathrm{D}$ is a distribution matrix used to allocate the data on the frequency grid called chip mapping matrix. Finally, $\mathrm{F}_{\mathrm{N}}$ is the Fourier matrix.

*Corresponding author: Nouri Naziha, Innov'COM Lab, Higher School of Communications of Tunis, Tunisia, Tel: (216)71874700; E-mail: nourinaziha@yahoo.fr

Received May 19, 2016; Accepted August 04, 2016; Published August 16, 2016

Citation: Naziha N, Bouallegue R (2016) Double Allocation of Spreading Code Minimizing SI and PAPR for LP-OFDM UWB System. J Telecommun Syst Manage 5: 137. doi:10.4172/2167-0919.1000137

Copyright: $\odot 2016$ Naziha N, et al. This is an open-access article distributed under the terms of the Creative Commons Attribution License, which permits unrestricted use, distribution, and reproduction in any medium, provided the original author and source are credited. 


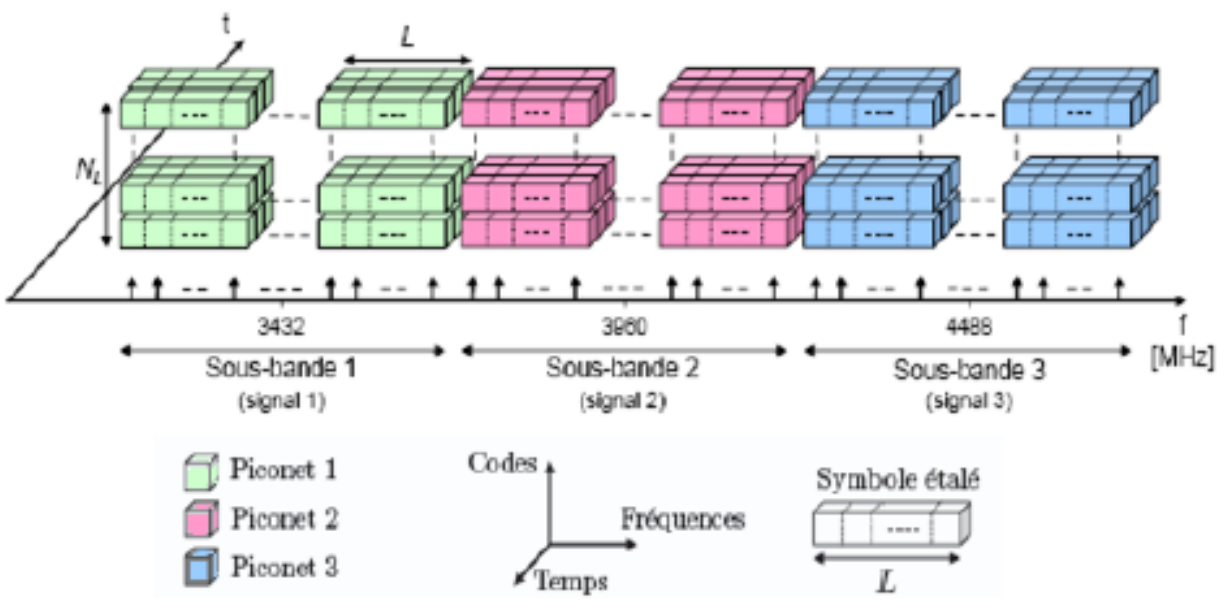

Figure 1: Chip mapping of multiblock LP- OFDM UWB system.

The matrix of output symbols of the OFDM modulator can be reformulated and becomes:

$$
S=F_{N}^{H} \cdot D \cdot\left[\begin{array}{lll}
C_{1} & & 0 \\
& C_{b} & \\
0 & & C_{B}
\end{array}\right]\left[\begin{array}{l}
X_{1} \\
X_{b} \\
X_{B}
\end{array}\right]
$$

where $B$ is the number of sub-blocks of subcarriers in the subband with $\mathrm{B} \times \mathrm{L}=\mathrm{N}$. $\mathrm{C}_{\mathrm{b}}$ is the matrix containing the $\mathrm{N}_{\mathrm{L}}$ pre-coding sequences of the sub-block $b(b \in[1 \ldots B]) . X_{b}$ is the matrix of $N_{L}$ vectors $N_{s}$ complex symbols transmitted in the sub-block $b$.

\section{Choice of Linear Precoding Matrix}

Codes Walsh-Hadamard is generated from the Sylvester- Hadamard transform matrix. They correspond to the rows or columns of the orthogonal matrix $(\mathrm{L} \times \mathrm{L})$ constructed recursively as follows:

$$
H_{L}=\left[\begin{array}{cc}
H_{L / 2} & H_{L / 2} \\
H_{L / 2} & -H_{L / 2}
\end{array}\right] \forall L=2^{n} n \in N^{*}, H_{1}=+1
$$

These Codes used in synchronous applications systems MultiCarrier Code Division Multiple Access (MC-CDMA) or LP-OFDM because of the facility to generate them. So we use these codes in the precoding matrix of the LP-OFDM.

The matrix of procoding $\mathrm{C}_{\mathrm{b}}$ will correspond to the $\mathrm{N}_{\mathrm{L}}$ columns of $\mathrm{H}_{\mathrm{L}}\left(\mathrm{N}_{\mathrm{L}}<=\mathrm{L}\right)$.

\section{Selection Criteria of Codes}

\section{Self-interference}

The choice of orthogonal codes for LP-OFDM system overcomes the SI. This is true if the transmission of the data is considered carried out on a Gaussian channel but it is not it any more if the transmission is carried out through a multipath channel. Indeed, in this case, the orthogonality between the spreading codes is broken by the distortions introduced by the multipath channel. It is then necessary to try to minimize this term SI at reception by the implementation of singleuser detection techniques.

\section{The PAPR and the crest factor (CF)}

The A multicarrier technique is a suitable solution for the transmission of signals over multipath channels. However, the amplitude of the signal LP-OFDM has strong fluctuations. The PAPR allows estimating the ratio between the power peak and the average power of the generated signal. The amplitude of the fluctuations is evaluated by the CF defined by:

$$
C F\left(S_{j}(t)\right)=\sqrt{\operatorname{PAPR}\left(S_{j}(t)\right)}=\sqrt{\frac{\text { puis.crete }\left(S_{j}(t)\right)}{\text { puis.moyenne }\left(S_{j}(t)\right)}}=\sqrt{\frac{\max \mid\left(\left.S_{j}(t)\right|^{2}\right.}{\frac{1}{T} \int_{0}^{T} \mid\left(\left.S_{j}(t)\right|^{2} d t\right.}}
$$

In practice, it is desired to transmit a signal with a maximum output power without distortion which leads to a degradation of system performance. The technique of selecting low crest factor sequences can remedy to this problem. Hence the interest is to search for OFDM systems the spreading codes with low PAPR value.

\section{Selection of Spreading Codes}

\section{Minimizing SI}

Estimate $y_{n, j}$ of the nth complex symbol $x_{n, j}$ of user $j$

$$
\hat{y}_{n, j}=x_{n, j} \sum_{l=1}^{L} c_{l}^{2} h_{1} \mathrm{~g}_{l}+\sum_{p=1}^{N_{L}} x_{p, j}+\sum_{l=1}^{L} c_{l, n} c_{l, p} h_{1} \mathrm{~g}_{l}+\sum_{l=1}^{L} c_{l, n} \mathrm{~g}_{l}
$$

Posing R $(k-l)=\varepsilon\left[h_{k} g_{k} h_{l} g_{l}\right]$, the power of SI associated with the complex symbol $n$ can be written as:

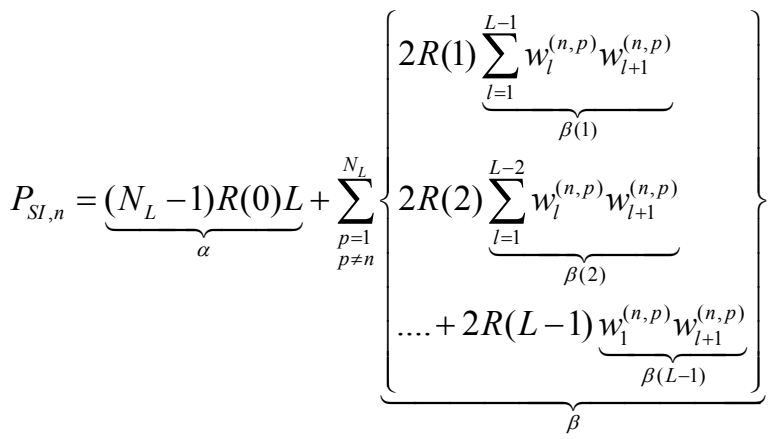

Or $\mathrm{w}_{1}{ }^{(\mathrm{n}, \mathrm{p})=} \mathrm{c}_{\mathrm{l}, \mathrm{n}} \mathrm{c}_{\mathrm{l}, \mathrm{p}}$ is defined as the product chip to chip of the spreading codes allocated to complex symbols $\mathrm{n}$ and $\mathrm{p}$ in the $\mathrm{l}$ th chip.

The power of the SI is influenced by the spreading codes and by selecting the used equalization technique. At full load, $\mathrm{N}_{\mathrm{L}}=\mathrm{L}$, the terms $\beta$ (i) are all negative and the same for each complex symbol. When $N_{L}<$ $\mathrm{L}$, the terms $\beta$ (i) are different depending on the used spreading codes. 
Some complex symbols are better over others where the power of the SI will be much more important. Therefore, minimizing the negative term $\beta$ (1) can reduce the power of the SI. This reduction is achieved by judiciously selecting the $\mathrm{N}_{\mathrm{L}}$ spreading codes to use.

The use of Walsh-Hadamard codes implies that $\mathrm{Nc}=\mathrm{L}$. Let's denote $\Omega$ the a spreading code family composed of Nc, $\Omega_{\mathrm{NL}}$ a subset of $\Omega$ compound $\mathrm{N}_{\mathrm{L}}$ Codes with $\mathrm{N}_{\mathrm{L}}<\mathrm{Nc}$. The $\mathrm{J}_{\Omega \mathrm{NL}}$ is define as a function such as:

$$
J^{\Omega N_{L}}=\max _{n, p \in \Omega N_{L}, n \neq p} I(n, p)
$$

Where $\mathrm{I}^{(\mathrm{n}, \mathrm{p})}$ is the function of interference produced by the sequence $\mathrm{p}$ on sequence $\mathrm{n}$. The $\mathrm{J}_{\Omega \mathrm{NL}}$ term takes into account the maximal degradation produced by two spreading sequences. The interference term $\mathrm{I}^{(\mathrm{n}, \mathrm{p})}$ is defined by:

$$
I^{(n, p)=}-T\left(W^{(n, p)}\right)
$$

where $\mathrm{W}^{(\mathrm{n}, \mathrm{p})}$ is a vector of $\mathrm{L}$ elements ${ }_{\mathrm{W} 1}{ }^{(\mathrm{n}, \mathrm{p})=} \mathrm{c}_{\mathrm{l}, \mathrm{n}} \mathrm{c}_{\mathrm{l}, \mathrm{p}}(l \in[1, \ldots$ , L]) resulting chip to chip produced between the $\mathrm{n}$ and $\mathrm{p}$ spreading sequences, $\mathrm{T}(\mathrm{v})$ defines the number of transitions between the elements of the vector $\mathrm{v}$ :

$$
T(v)=\frac{1}{2} \sum_{l=1}^{L-1}\left|\operatorname{sgn}() v_{i+1}-\operatorname{sgn}\left(v_{i}\right)\right|
$$

Thus, the minimization $\mathrm{J}_{\Omega \mathrm{NL}}$ to preserve a subset of $\mathrm{N}_{\mathrm{L}}$ sequences for which the various vectors $\mathrm{W}^{(\mathrm{n}, \mathrm{p})}$ presents a maximum number of transitions. The selection of the optimum subset of spreading sequences is thus defined by:

$$
\Omega_{N_{L}}^{(\text {opt })}=\arg \min _{\Omega N_{L} \in \Omega} J^{\left(\Omega N_{L}\right)}
$$

So this method can widely select the spreading codes to obtain an optimal system LP-OFDM. According to the family of spreading codes used, it may happen that the selection process leads to obtain more optimal subsets, the latter having equal $J \Omega N L$ functions. Under these conditions, it is possible to arbitrarily choose one of the optimal subsets or use Complementary selection criterion.

Complementary criterion: MEAN: This complementary criterion consists to minimize the average number of transitions between the different terms $\mathrm{I}^{(\mathrm{n}, \mathrm{p})}$ of the same group $\mathrm{N}_{\mathrm{L}}$ minimizing a new function defined as:

$$
{ }_{\text {moy }}^{\left(\Omega N_{L}\right)}=\underset{n \text { et } p \in \Omega N_{L} n \neq p}{\operatorname{moy}} I^{(n, p)}
$$

Complementary criterion: STD: The principle of this complementary criterion nearly identical to the previous. It is defined by:

$$
J_{S_{T D}}^{\left(\Omega N_{L}\right)}=\underset{n \text { et }}{\operatorname{std} \in \Omega N_{L} n \neq p} I^{(n, p)}
$$

Where std (v) represents the function standard deviation of $\mathrm{v}$. This criterion consists in minimizing the standard deviation amongst transitions between various terms $\mathrm{I}^{(\mathrm{n}, \mathrm{p})}$ in the same group $\mathrm{N}_{\mathrm{L}}$.

Complementary criterion: 2nd order: The principle of this complementary criterion is an extension of the first criterion. Indeed, this criterion searches to maximize the minimum number of transitions within the vectors:

$$
w^{\prime(n, p)}=w_{1}^{(n, p)}, w_{3}^{(n, p)}, \ldots . w_{L-3}^{(n, p)}, w_{L-1}^{(n, p)}
$$

and

$$
w^{n(n, p)}=w_{2}^{(n, p)}, w_{4}^{(n, p)}, \ldots . . w_{L-2}^{(n, p)}, w_{L}^{(n, p)}
$$

\section{Minimizing Joint SI and PAPR}

The algorithm of minimization joint power SI and PAPR or peak factor is made by steps detailed as follows:

- Step 1: Select the best complementary selection criteria whether the standard deviation : STD or The second order : $2^{\text {nd }}$ order

- Step 2: Apply the complementary selection criteria already chosen earlier can get more optimal subsets

- Step 3: Calculate the optimal PAPR for each subset

- Step 4: Select the optimal subset having the minimum PAPR.

\section{Simulation Results}

The performance are given for the simulation chain LP-OFDM in the case of a coding rate $\mathrm{R}=1 / 2$ and a minimum mean square error (MMSE) detection on the channel CM1.

Figure 2 shows the performance obtained in the case of misallocation and in the case of an optimal allocation of spreading codes. The length of the codes of Walsh-Hadamard used is $\mathrm{L}=16$, the system performs with the loads $\mathrm{N}_{\mathrm{L}}=8$ and $\mathrm{N}_{\mathrm{L}}=15$. In conclusion that for $\mathrm{N}=8$, a bad selection of spreading codes can significantly degrade system performance. So, it is important to correctly select the codes for optimal operation of the system LP-OFDM. This degradation is very low in contrast to $\mathrm{N}_{\mathrm{L}}=15$. A good selection of spreading codes is even better than the load is low. When $\mathrm{N}_{\mathrm{L}}$ increases to $\mathrm{L}$, the difference in performance with a good selection and a bad selection attenuates to the limited case where $\mathrm{N}_{\mathrm{L}}=\mathrm{L}$.

In the condition, the complementary selection criterion is used for obtaining more optimal subsets. The Performance Comparison of these criteria is illustrated in Figure 3 in order to extract the most optimal. For a $\mathrm{BER}=10^{-3}$ the gain is $\mathrm{Eb} / \mathrm{No}=0.66 \mathrm{~dB}$ in the case of the criterion of the standard deviation and second order but the gain is reduced to $\mathrm{Eb}$ / $\mathrm{No}=0.33 \mathrm{~dB}$ for the case of the mean criterion. So, According to this figure 3, they are the two criteria which are more optimal: the criterion of the standard deviation and the criterion of the second order.

Figure 4 shows a comparison of performance between an optimum

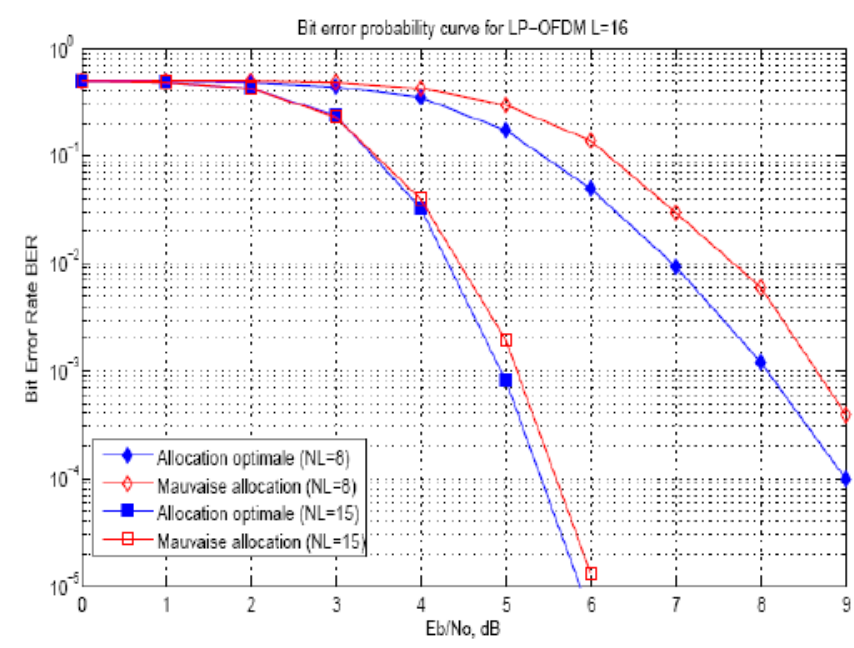

Figure 2: Example LP-OFDM system performance comparison in the case of a good and bad selection of the spreading codes obtained with $L=16, N_{L}=8$ and $\mathrm{N}_{\mathrm{L}}=15$. 


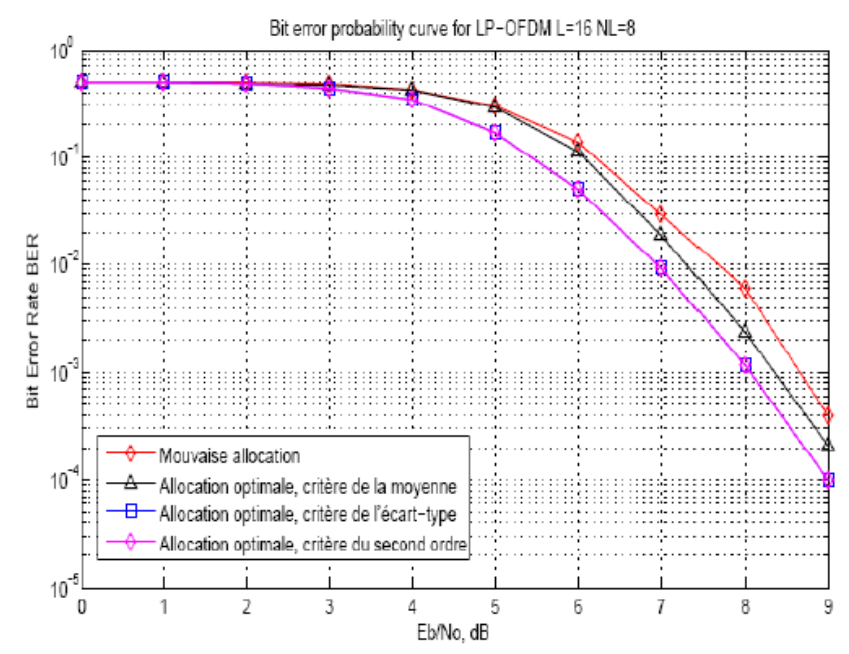

Figure 3: Performance Comparison of LP-OFDM system in the case of misallocation and optimal complementary selection criteria of the spreading codes obtained with $L=16, N_{L}=8$.

selection using the second order criterion, minimizing SI only and the second criterion minimizing PAPR.

This dual minimization is applied to different load $\mathrm{N}_{\mathrm{L}}$. It shows an improvment in performance compared to the conventional criterion. Indeed, the Figure 4 shows an acceptable and variable gain according to load $\mathrm{N}_{\mathrm{L}}$. The best profit is shown for the low load $\mathrm{N}_{\mathrm{L}}<\mathrm{L}$ but when $\mathrm{N}_{\mathrm{L}}$ increases towards $\mathrm{L}$, the performance difference decreases until the limiting case where $\mathrm{N}_{\mathrm{L}}=\mathrm{L}$ because SI tends towards 0 . Consequently, the optimal subsets are equal. In this case the application of PAPR has no influence and the performance is achieved its best for full load.

\section{Conclusion}

In this paper, a novel method has proposed which consists of a double selection of spreading code. It can reduce jointly the SI power and the peak factor of the signal. This method show more efficient in terms of performance compared to the conventional method which uses three complementary criterions minimizing only the SI power when the system does not operate with full load.

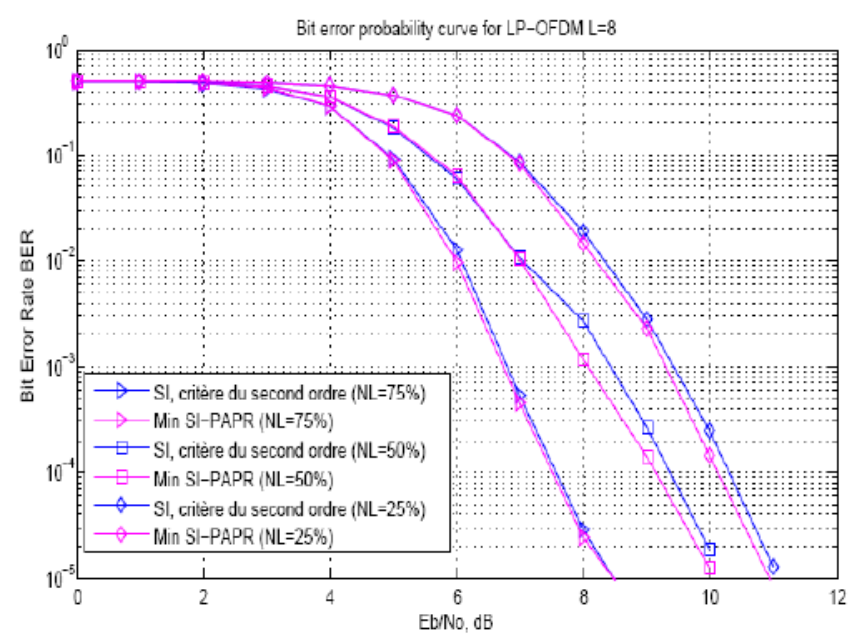

Figure 4: LP-OFDM system performance comparison in the case of an optimal allocation of complementary criterion of second order and joint SI and PAPR minimization obtained with $\mathrm{L}=8, \mathrm{NL}=2, \mathrm{NL}=4$ et $\mathrm{NL}=6$.

\section{References}

1. Hara S, Prasad R (1997) Overview of multicarrier CDMA. IEEE Communications Magazine 35: 26-133.

2. Weinstein SB (2009) the history of orthogonal frequency-division multiplexing IEEE Communications Magazine 47: 26-35.

3. Baudais JY (2012) Une contribution à l'allocation des ressources des systèmes précodés à porteuses multiples. Habilitation à diriger des recherches Spécialité traitement du signal Rapport d'activite.

4. Muhammad FS, Baudais JY, Hélard JF (2009) Rate maximization loading algorithm for LP-OFDM systems with imperfect CSI. 20th Personal Indoor and Mobile Radio Communications, Tokyo pp.1-5.

5. Antoine S (2008) Resource Allocation Strategies and Linear Precoded OFDM Optimization for Ultra-Wideband Communications.

6. Fazel K, Kaiser S (2003) Multi-Carrier and Spread Spectrum Systems. John Wiley \& Sons

7. Nobilet S, H'elard JF, Mottier D (2002) Spreading sequences for uplink and downlink MC-CDMA systems: PAPR and MAI minimization. European Transactions on Telecommunication 13: 465-474. 